\title{
Primary research \\ Comparison of written reports of mammography, sonography and magnetic resonance mammography for preoperative evaluation of breast lesions, with special emphasis on magnetic resonance mammography
}

\author{
Sabine Malur*, Susanne Wurdinger ${ }^{\dagger}$, Andreas Moritz*, Wolfgang Michels* and Achim Schneider*
}

*Department of Gynecology, Friedrich-Schiller University, Jena, Germany

${ }^{\dagger}$ Institute for Diagnostic and Interventional Radiology, Friedrich-Schiller University, Jena, Germany

Correspondence: Achim Schneider MD, MPH, Professor and Chairman, Department of Gynecology, Friedrich-Schiller University, Bachstraße 18, 07740 Jena, Germany. Tel: +49 3641933 063; fax: +49 3641933 064; e-mail: aschneider@med.uni-jena.de

Received: 12 April 2000

Breast Cancer Res 2001, 3:55-60

Revisions requested: 22 May 2000

Revisions received: 24 June 2000

Accepted: 5 October 2000

(C) BioMed Central on behalf of the copyright holder

Published: 2 November 2000

(Print ISSN 1465-5411; Online ISSN 1465-542X)

\begin{abstract}
Patients with abnormal breast findings $(n=413)$ were examined by mammography, sonography and magnetic resonance (MR) mammography; 185 invasive cancers, 38 carcinoma in situ and 254 benign tumours were confirmed histologically. Sensitivity for mammography was $83.7 \%$, for sonography it was $89.1 \%$ and for MR mammography it was $94.6 \%$ for invasive cancers. In 42 patients with multifocal invasive cancers, multifocality had been detected by mammography and sonography in $26.2 \%$, and by MR mammography in $66.7 \%$. In nine patients with multicentric cancers, detection rates were 55.5 , 55.5 and $88.8 \%$, respectively. Carcinoma in situ was diagnosed by mammography in $78.9 \%$ and by MR mammography in $68.4 \%$ of patients. Combination of all three diagnostic methods lead to the best results for detection of invasive cancer and multifocal disease. However, sensitivity of mammography and sonography combined was identical to that of MR mammography (ie 94.6\%).
\end{abstract}

Keywords: breast lesions, diagnostic methods

\section{Introduction}

Mammography and sonography are the standard imaging techniques for detection and evaluation of breast disease [1]. Mammography is the most established screening modality [2]. Especially in young women and women with dense breasts, sonography appears superior to mammography, and differentiation between solid tumours and cysts is easier. Sensitivity and specificity of sonography or mammography are higher if sonography and mammography are combined [3].
It is generally accepted that MR mammography is the most sensitive technique for diagnosis of breast cancer, whereas the reported specificity of MR mammography varies [4-12]. In those studies, MR mammography was performed and evaluated by highly specialized radiologists in a research setting. It was therefore the purpose of the present prospective study to compare the validity of MR mammography with mammography and sonography in clinical routine practice. Findings for the three diagnostic methods documented on routine reports that

FFE $=$ fast-field-echo; $M R=$ magnetic resonance. 
were available to the surgeon preoperatively formed the basis of this comparison. Special emphasis was placed on the identification of multifocal and multicentric invasive disease.

\section{Patients and methods Patients}

Between September 1995 and September 1998, 413 patients with abnormal breast findings were referred for histological evaluation to the Department of Gynecology of the Friedrich-Schiller University, Jena, Germany. Patients had been selected and referred because of the presence of breast lesions detected by palpation and/or mammography and/or sonography. In addition, MR mammography was performed in all patients. We excluded five patients with invasive cancer who had a history of coreneedle or fine-needle biopsy cancer within 2 weeks before referral, because the presence of haematoma may mimic false-positive findings on MR mammography. In addition, five patients who did not keep still during MR mammography were excluded.

\section{Imaging}

Analysis of the sonograms taken in patients with histologically confirmed carcinoma in situ were excluded from analysis because the value of sonography for detection of premalignant disease is limited. Mammography was not performed in 32 patients who were younger than 30 years or who had had a mastectomy with suspected local recurrence. For all patients, written reports of mammographic, sonographic and dynamic MR mammographic findings were available preoperatively.

The majority of mammograms $(68 \%)$ were performed at the Institute for Diagnostic and Interventional Radiology, Friedrich-Schiller-University, using a senograph DMR (GE Medical Systems, Milwaukee, Wisconsin, USA) with standard craniocaudal and mediolateral oblique projections. Mammograms obtained at other institutions that were considered to match the quality standards of our institution were also accepted for evaluation.

All sonography and MR examinations were carried out at the Department of Gynecology and the Institute for Diagnostic Interventional Radiology, Friedrich-Schiller-University, respectively. Sonography was done using a $7.5-\mathrm{MHz}$ linear array probe with a Sonoline Versa Pro (Siemens, Erlangen, Germany). MR mammography was performed exclusively at the Institute for Diagnostic Interventional Radiology, using a Gyroscan ACS II (Philips, Nijmegen, The Netherlands) with a field strength of $1.5 \mathrm{~T}$ using a double-breast coil. Dynamic T1-weighted images were aquired using a multislice twodimensional fast-field-echo (FFE) sequence. We used the following parameters: TR 97, TE 5.0, flip angle $80^{\circ}$, slice thickness $4.0 \mathrm{~mm}$, gap $0.4 \mathrm{~mm}$, field of view $350 \mathrm{~mm}$ and transverse orientation. In addition T2-weighted images $\left(4000 / 300 / 90^{\circ} / 4.0 \mathrm{~mm} / 0.4 / 350 \mathrm{~mm}\right)$ were obtained. As contrast medium, $0.1 \mathrm{mmol}$ gadolinium-DTPA $/ \mathrm{kg}$ body weight (Magnevist; Schering, Berlin, Germany) was used and injected as a bolus. One unenhanced and seven enhanced studies were acquired with an acquisition time of $1 \mathrm{~min}$. Criteria for malignancy were signal enhancement of $90 \%$ or more within the first 2 min after bolus injection and signal plateau or washout phenomena afterward. Additional criteria were irregular borders of the lesion and low signal intensity in the T2-weighted images.

Mammograms were read by three different radiologists, sonography was done by three different gynaecologists and MR mammography was interpreted by a total of six different radiologists.

Definition for multifocal carcinoma was a distance of less than $3 \mathrm{~cm}$ and for multicentric carcinoma a distance over $3 \mathrm{~cm}$ between various lesions.

\section{Statistical analysis}

Interpretation of the various diagnostic procedures was compared with the histological examination with regard to sensitivity, specificity, accuracy, and positive and negative predictive value. Criteria for suspected malignancy in the written reports were the terms 'cancer', 'malignant lesion or tumor', or 'suspicious for cancer'. Sensitivity, specificity, negative and positive predicitive value, and accuracy were evaluated as follows:

Sensitivity $=$ patients with suspected breast cancer/ patients with histologically confirmed breast cancer

Specificity $=$ patients with suspected benign disease/ patients with histologically confirmed benign disease

Positive predictive value $=$ patients with histologically confirmed breast cancer/patients with suspected breast cancer

Negative predictive value $=$ patients with histologically confirmed benign disease/patients with suspected benign disease

Accuracy $=$ patients with true-positive and true-negative detected disease/patients with histologically confirmed breast cancer

A result was classified as false-negative when a diagnostic method classified a histologically confirmed cancer as benign. A result was classified as false-positive when a diagnostic method classified a histologically confirmed benign lesion as cancer. We compared the preformance of all diagnostic methods individually and in combination using the results from all patients. Statistical analysis was performed for all variables with Fisher's exact test and Pearson's $\chi^{2}$ test. 


\section{Results}

All patients underwent breast surgery and all abnormal lesions identified by mammography, sonography or MR mammography were surgically removed. A total of 477 breast lesions were examined histologically, revealing the presence of 185 invasive cancers, 38 carcinomata in situ and 254 benign lesions (fibroadenoma, papilloma, intraductal or adenoid ductal hyperplasia, cystic mastopathia). There were four patients with malignant lesions in both breasts. In 42 patients multifocal tumours and in nine patients multicentric tumors were found on histological examination. Among the 185 invasive lesions, 178 were primary cancers, five were recurrences, one was metastatic and one was an angiosarcoma. The majority of invasive breast cancers were staged as pT1c (44\%). Six per cent of tumors were detected in stage pT1a, $18 \%$ in stage pT $1 \mathrm{~b}, 25 \%$ in stage pT2, $3 \%$ in stage pT3 and $4 \%$ in stage pT4. The distribution of histopathological tumour types is shown in Table 1. The mean age of patients was 58 years (range $19-85$ years).

The sensitivity of MR mammography was significantly higher than those of mammography and sonography $(P<0.005$ and $P<0.05$; Table 2$)$. The specificity of sonography was significantly higher than those of mammography and MR mammography $(P<0.05$ and $P<0.005$; Table 2). The negative predictive values for sonography and MR mammography were significantly higher than that of mammography $\quad(P<0.05$ and $P<0.005$; Table 2). With regard to accuracy, no significant difference between the three modalities was found (Table 2). Combining of all three diagnostic methods yielded the best results for detection of cancer $(P<0.005$; Table 3). The sensitivity and negative predictive value for the combination of mammography and MR mammography, and the combination of sonography and MR mammography were significantly higher than those for the combination of mammograpy and sonography $(P<0.05$; Table 3$)$. The highest result for accuracy was seen for a combination of all three methods $(P<0.05$; Table 3$)$.

Mammography was false-negative in 30 out of 184 invasive cancers, sonography was false-negative in 20 out of 185 cancers, and 10 out of 185 invasive cancers were missed by MR mammography. The majority of false-negative findings was found in stage 1 disease, ductal carcinoma and grade 3 tumors (Table 4). Of 10 invasive cancers missed by MR mammography, eight were found by mammography and sonography. By all three techniques, one invasive ductal carcinoma (pT1b) was misinterpreted as fibroadenoma. In another patient, a microinvasive lobular carcinoma of $5 \mathrm{~mm}$ diameter was not detected with mammography and MR mammography, whereas sonography detected a solid, benign tumour. MR mammography identified 10 invasive cancers $(5.2 \%)$ that were missed by mammography and sonography, whereas one invasive cancer was found
Table 1

\begin{tabular}{lc} 
Histopathology of $\mathbf{1 8 5}$ invasive cancers & \\
\hline Histopathologic diagnosis & Number of lesions \\
\hline Ductal carcinoma & 109 \\
Lobular carcinoma & 36 \\
Mixed ductal/lobular carcinoma & 16 \\
Mucinous carcinoma & 2 \\
Medullary carcinoma & 3 \\
Tubular carcinoma & 13 \\
Others (metaplastic carcinoma, & 6 \\
$\quad$ angiosarcoma, metastases) & \\
\hline
\end{tabular}

by mammography alone. By sonography alone, not a single case of invasive disease was detected when MR mammography or mammography were nonsuspected.

The highest detection rate for multifocal invasive disease was seen with MR mammography, which identified 28 out of $42(66.7 \%)$ histologically confirmed multifocal invasive cancers, whereas mammography and sonography both identified $11(26.2 \%)$ of these cancers $(P<0.05)$. The combination of all three diagnostic methods leads to the best result for detection of multifocality $(76.2 \% ; P<0.05)$, whereas the detection rate with the combination of mammography and sonography was $35.7 \%$, with the combination of sonography and MR mammography it was $69 \%$ ( $P<0.05$ versus mammography + sonography), and with the combination of mammography and MR mammography it was $73.8 \%(P<0.05$ versus mammography + sonography). Multifocal invasive disease was suspected in 12 patients by mammography, in 13 patients by sonography, and in 16 patients by MR mammography, but only unifocal disease was confirmed by histology. Out of nine patients with histologically confirmed multicentric invasive cancer, eight $(88.8 \%)$ of these cancers were detected by MR mammography and five (55.5\%) by mammography or sonography. One patient was diagnosed with multicentric invasive-lobular carcinoma stage pT2G2, which had been misinterpreted as benign tumour by sonography and mammography, and as haematoma by MR mammography.

Out of 38 patients with carcinoma in situ, mammography (suspicious microcalcifications, exclusively) identified 30 cases (78.9\%) and MR mammography identified 26 cases (68.4\%). When combining mammography and MR mammography, sensitivity for detection of carcinoma in situ increased to $87 \%$ (not significant).

\section{Discussion}

When the validity of individual diagnostic methods for detection of invasive breast cancer was analyzed, the 
Table 2

Comparison of mammography, sonography and MR mammography for preoperative prediction of histologically confirmed invasive cancer $(n=185)$ or benign disease $(n=254)$

\begin{tabular}{lccccc}
\hline Diagnostic method & Sensitivity (\%) & Specificity (\%) & PPV (\%) & NPV (\%) & Accuracy (\%) \\
\hline Mammography & 83.7 & 68.5 & 67.8 & 84.1 & 77.1 \\
Sonography & 89.1 & $79.1^{\star \dagger}$ & 65.7 & $90.9^{\ddagger}$ & $83.4^{\dagger}$ \\
MR mammography & $94.6^{\star \ddagger}$ & 68.5 & 68.6 & $94.6^{\dagger}$ & 79.5 \\
\hline
\end{tabular}

${ }^{\star} P<0.005$, versus MR mammography; ${ }^{\dagger} P<0.05$, versus mammography; ${ }^{\ddagger} P<0.05$, versus sonography. NPV, negative predictive value;

PPV, positive predictive value.

Table 3

Comparison of various combinations of three diagnostic methods for preoperative prediction of histologically confirmed invasive cancer $(n=185)$ or benign disease $(n=254)$

\begin{tabular}{lccccc}
\hline Diagnostic methods & Sensitivity (\%) & Specificity (\%) & PPV (\%) & NPV (\%) & Accuracy (\%) \\
\hline Mammography + MR mammography & $98.9^{*}$ & 90.5 & 88.4 & $99.1^{*}$ & 94.1 \\
Sonography + MR mammography & $98.9^{*}$ & 93.3 & 91.5 & $99.2^{*}$ & 95.7 \\
Mammography + sonography & 94.6 & 92.1 & 89.7 & 95.9 & 93.2 \\
All three methods combined & $99.4^{\dagger}$ & 95.3 & 93.9 & $99.6^{\dagger}$ & $97.0^{\dagger \neq}$ \\
\hline
\end{tabular}

${ }^{\star} P<0.05$, versus mammography and sonography; ${ }^{\dagger} P<0.005$, versus mammography and sonography; ${ }^{\ddagger} P<0.05$, versus MR mammography and mammography. NPV, negative predictive value; PPV, positive predictive value.

Table 4

\begin{tabular}{|c|c|c|c|}
\hline $\begin{array}{l}\text { Cancer } \\
\text { characteristics }\end{array}$ & Mammography & Sonography & $\begin{array}{c}\text { MR } \\
\text { mammography }\end{array}$ \\
\hline \multicolumn{4}{|l|}{ Tumour stage } \\
\hline $1 \mathrm{a}$ & 1 & 4 & 3 \\
\hline $1 b$ & 8 & 5 & 2 \\
\hline $1 \mathrm{c}$ & 11 & 6 & 3 \\
\hline 2 & 6 & 2 & 2 \\
\hline 3 & 1 & 1 & 0 \\
\hline 4 (inflammatory) & 3 & 2 & 0 \\
\hline \multicolumn{4}{|l|}{ Histological type } \\
\hline Ductal & 15 & 13 & 2 \\
\hline Lobular & 11 & 3 & 4 \\
\hline Others & 4 & 4 & 4 \\
\hline \multicolumn{4}{|l|}{ Grading } \\
\hline 1 & 2 & 3 & 1 \\
\hline 2 & 10 & 6 & 5 \\
\hline 3 & 18 & 11 & 4 \\
\hline
\end{tabular}

sensitivity and specificity of mammography ranged from 79.9 to $89 \%$ and from 64 to $93.5 \%$, respectively $[6,7,13]$; for sonography from 67.6 to $96 \%$ and from 93 to $97.7 \%$, respectively [13,14]; and for MR mammography from 91 to 98.9 and from 20 to $97.4 \%$, respectively [6-10, 15-17].
The performance of mammography, sonography and MR mammography was compared in three large series (Table 5). The present results are similar with regard to sensitivity and specificity for the detection of malignant breast lesions, with MR mammography reaching the highest sensitivity of all imaging procedures.

When combinations of all three technique were analyzed, mammography and sonography (standard method) had a sensitivity of $83 \%$ and a specificity of $92 \%$ for detection of malignant disease [3]. A combination of mammography, sonography and MR mammography (combined method) showed a sensitivity of $95 \%$ and a specificity of $64 \%$ [3]. For nonpalpable lesions, sensitivity increased from $73 \%$ by the standard method to $82 \%$ for the combined method. Specificity for the standard method (89\%) was higher than that for the combined method (71\%). For palpable lesions a sensitivity of $85 \%$ for the standard and $98 \%$ for the combined method was achieved, whereas specificity for the standard method was 100\% compared with $45 \%$ for the combined methods [3]. The positive predictive value was $94 \%$ for the standard and $80 \%$ for the combined methods, and the negative predictive values were 78 and $89 \%$, respectively [3]. In the present study, we also found the highest sensitivity, specificity, and positive and negative predictive values for the combination of all three methods. Combination of mammography and sonography was as sensitive as MR mammography alone (94.6\% versus $94.6 \%$ ). 
Table 5

Review of the literature: case series that compared mammography, sonography and MR mammography in patients with benign tumours and invasive cancer

\begin{tabular}{|c|c|c|c|c|c|c|c|}
\hline \multirow[b]{2}{*}{ Reference } & \multirow[b]{2}{*}{$n$} & \multicolumn{2}{|c|}{ Mammography } & \multicolumn{2}{|c|}{ Sonography } & \multicolumn{2}{|c|}{ MR mamography } \\
\hline & & Sens $(\%)$ & Spec (\%) & Sens (\%) & Spec (\%) & Sens (\%) & Spec (\%) \\
\hline [29] & 60 & 82 & 96 & 79 & 50 & 95 & 64 \\
\hline [14] & 150 & 88 & 88 & 96 & 93 & 98 & 99 \\
\hline [30] & 98 & 91 & - & 83 & - & 98 & - \\
\hline
\end{tabular}

-, not indicated; Sens, sensitivity; Spec, specificity.

The majority of false-positive results for invasive cancer by MR mammography (80 out of 439) were caused by papillomas, intraductal hyperplasia grade 2 or 3 , or fibroadenomas in the present series. These lesions have a good blood supply and may mimic invasive cancer $[16,18]$.

Ten out of 185 (5.4\%) malignant lesions were classified as false-negative by MR mammography. On histology, the majority of false-negative invasive cancers were lobular cancers (four out of 10). Bone et al [18] reported falsenegative results in 11 out of 155 readings, with the majority being lobular cancers on histology. Lack of tumour-induced neovascularity may explain such findings. In particular, invasive lobular cancers infiltrate the normal tissue with columns of single cells, and receive adequate oxygenation without the requirement for increased vascularization [19]. Buadu et al [11] found that lobular and mucinous carcinomas had a low microvessel density.

Multifocality of breast cancers can be recognized adequately by MR mammography $[20,21]$. Boetes et al [22] reported that all 61 multifocal cancers were detected by MR mammography, compared with $31 \%$ by mammography and $38 \%$ by sonography. Esserman et al [20] detected multifocality by MR mammography in 100\% (10 out of 10 ) versus $44 \%$ (four out of nine) by mammography. Relevant changes in therapy due to additional multicentric and contralateral tumour findings by MR mammography occur in $18 \%$ of patients as compared with conventional imaging [23]. We found a detection rate of multifocality of $66.7 \%$ by MR mammography, as compared with $26.2 \%$ by mammography and sonography. However, in 16 patients multifocal invasive disease as diagnosed by MR mammography was shown to be unifocal by histology.

Kramer et al [24] reported that MR mammography yielded the highest sensitivity for detection of multicentricity as compared with mammography and sonography $(89,66$ and $79 \%$, respectively) in 38 patients. These findings are comparable with the present results, in which eight out of nine multicentric cancers were diagnosed correctly.
Carcinoma in situ is identified by mammography through the presence of suspicious microcalcifications. Suspicious microcalcifications are more frequent in intraductal than in infiltrating cancers [25], which was also observed in the present series. Mammography showed a detection rate for carcinoma in situ of $78.9 \%$, as compared with $65.8 \%$ by MR mammography; the combination of mammography and MR mammography lead to a detection rate of $86.4 \%$. Fischer et al [26] reported that carcinoma in situ was identified by MR mammography in 25 out of 35 patients (72\%); three ductal carcinomata in situ were detected by MR mammography exclusively. Sittek et al [27] reported that 14 out of 20 carcinomata in situ (70\%) were correctly diagnosed by MR mammography on the basis of focal increase of signal intensity. Those authors concluded that carcinoma in situ is not reliably detected by MR mammography because of lack of a uniform pattern of enhancement. Esserman et al [20] reported a detection rate of $43 \%$ for ductal carcinoma in situ by MR mammography. Among 36 woman with carcinoma in situ, Gilles et al [28] demonstrated two cases without early contrast enhancement.

The present study showed that, for detection of breast cancer, MR mammography is not superior to a combination of sonography and mammography. For identification of multifocal or multicentric disease, MR mammography proved to be the most accurate technique.

\section{References}

1. Orel SG, Troupin RH: Nonmammographic imaging of the breast: current issues and future prospects. Semin Roentgenol 1993, 28:231-241.

2. Kerlikowske K, Grady D, Rubin SM, Sandrock C, Ernster VL: Efficancy of screening mammography. JAMA 1995, 273:149-154.

3. Müller-Schimpfle M, Stoll P, Stern W, Kutz S, Dammann F, Claussen CD: Do mammography, sonography and MR mammography have a diagnostic benefit compared with mammography and sonography? AJR Am J Roentgenol 1997, 168:1323-1329.

4. Heywang SH, Hahn D, Schmidt $H$, Krischke I, Eiermann W, Bassermann R, Lissner J: MR imaging of the breast using gadolinium-DTPA. J Comput Assist Tomogr 1986, 10:199-204.

5. Kaiser WA, Zeitler E: MR mammography: first clinical results [in German]. Röntgenpraxis 1985, 38:256-262.

6. Kacl GM, Liu P, Debatin JF, Garzoli E, Caduff RF, Krestin GP: Detection of breast cancer with conventional mammography and contrast-enhanced MR imaging. Eur Radiol 1998, 8:194200. 
7. Bone B, Pentek Z, Perbeck L, Veress B: Diagnostic accuracy of mammography and contrast-enhanced MR imaging in 238 histologically verified breast lesions. Acta Radiol 1997, 38: 489-496.

8. Kaiser WA: MR Mammographie. Radiologe 1993, 33:292-299.

9. Heywang-Köbrunner SH: Contrast-enhanced magnetic resonance imaging of the breast. Invest Radiol 1994, 29:94-104.

10. Harms SE, Flaming DP: MR imaging of the breast. J Magn Reson Imaging 1993, 3:277-283.

11. Buadu LD, Murakami J, Murayama S, Hashiguchi N, Sakai S, Masuda K, Toyoshima S: Breast lesions: correlation of contrast medium enhancement patterns on MR images with histopathologic findings and tumor angiogenesis. Radiology 1996, 200:639-649.

12. Orel SG, Schnall MD, LiVolsi VA, Troupin RH: Suspicious breast lesions: MR imaging with radiologic-pathologic correlation. Radiology 1994, 190:485-493.

13. Ciatto S, Rosselli del Turco M, Catarzi S, Morrone D: The contribution of sonography to the differential diagnosis of breast cancer. Neoplasma 1994, 41:341-345.

14. Oh KK, Cho JH, Yoon CS, Jung WH, Lee HD: Comparative studies of breast diseases by mammography, ultrasonography and contrast-enhanced dynamic magnetic resonance imaging. In: Breast Ultrasound Update. Edited by Madjar $\mathrm{H}$, Teubner J, Hackelöer BJ. Basel: Karger; 1994:332-343.

15. Heywang SH: MRI in breast disease. In: Book of Abstracts: Society of Magnetic Resonance in Medicine 1. Berkeley, CA. 1993:abstract 113.

16. Kaiser WA: False-positive results in dynamic MR mammography. Causes, frequency and methods to avoid. Magn Reson Imaging Clin North Am 1994, 2:539-555.

17. Heywang-Koebrunner $\mathrm{SH}$, Vieweg $\mathrm{P}$, Heinig $A$, Kuchler C: Contrast-enhanced MRI of the breast: accuracy, value, controversies, solutions. Eur J Radiol 1997, 24:94-108.

18. Bone B, Aspelin P, Bronge L, Isberg B, Perbeck L, Veress B: Sensitivity and specificity of MR mammography with histopathological correlation in $\mathbf{2 5 0}$ breasts. Acta Radiol 1996, 37:208-213.

19. Stelling CB: MR imaging of the breast for cancer evaluation: current status and future directions. Radiol Clin North $\mathrm{Am}$ 1995, 33:1187-1204.

20. Esserman L, Hylton N, Yassa L, Barclay J, Frankel S, Sickles E: Utility of magnetic resonance imaging in the managment of breast cancer: evidence for improved preoperative staging. $J$ Clin Oncol 1999, 17:110-119.

21. Kaiser WA, Diedrich K, Reiser M, Krebs D: Modern diagnostics of the breast [in German]. Geburtsh u Frauenheilk 1993, 53: $1-14$.

22. Boetes C, Mus R, Holland R, Barentsz JO, Strijk SP, Wobbes T, Hendriks JH, Ruys SH: Breast tumors: comparative accuracy of MR imaging relative to mammography and US for demonstration extent. Radiology 1995, 197:743-747.

23. Fischer U, Vossheinrich R, Kopka L, von Heyden D, Oestmann JW, Grabbe EH: Preoperative MR mammography in patients with breast cancer: impact of therapy [abstract]. Radiology 1994, 193:121.

24. Kramer S, Schultz-Wendtland R, Hagedorn K, Bautz W, Lang N: Magnetic resonace imaging and its role in the diagnosis of multicentric breast cancer. Anticancer Res 1998, 18:2163-2164.

25. Ciatto S, Rosselli del Turco M, Bonardi R, Cataliotta L, Distante V, Cardona G, Bianchi S: Non-palpable lesions of the breast detected by mammography: review of 1182 consecutive histologically confirmed cases. Eur J Cancer 1994, 30A:40-44.

26. Fischer U, Westerhof JP, Brinck U, Korabiowska M, Schauer A Grabbe E: Ductal carcinoma in situ in dynamic MR mammography at 1.5 T [in German]. Fortschr Röntgenstr 1996, 164: 290-294.

27. Sittek H, Kessler M, Heuck AF, Bredl T, Perlet C, Künzer I, Lebeau A, Untch M, Reiser M: Morphology and contrast enhancement of ductal carcinoma in situ in dynamic 1.0 T MR mammography [in German]. Fortschr Röntgenstr 1997, 167:2247-2251.

28. Gilles R, Zafrani B, Guinebretiere JM, Meunier M, Lucidarme $O$ Tardivon AA, Rochard F, Vanel D, Neuenschwander S, Arriagada $\mathrm{R}$ : Ductal carcinoma in situ: MR imaging-histopathologic correlation. Radiology 1995, 196:415-419.

29. Buchberger W, Kapferer M, Stöger A, Chemelli A, Judmaier W, Felber S: Dignity evaluation of focal mamma lesions: a prospective comparison between mammography, sonography and dynamic MR mammography [in German; abstract]. Radiologe 1995, 35:86.

30. Heywang SH, Wolf A, Pruss E, Hilbertz T, Eiermann W, Permanetter W: MR imaging of the breast with Gd-DTPA: use and limitations. Radiology 1989, 171:95-103. 